\title{
WIDESPREAD ATYPICAL CUTANEOUS LEISHMANIASIS CAUSED BY LEISHMANIA (L.) CHAGASI IN NICARAGUA
}

\author{
ALEJANDRO BELLI, DAVID GARCÍA, XIOMARA PALACIOS, BETZABÉ RODRIGUEZ, SONIA VALLE, \\ ELSA VIDEA, ENA TINOCO, FRANCISCA MARÍN, AND EVA HARRIS \\ Infectious Diseases Unit, Division of Public Health Biology and Epidemiology, School of Public Health, University of California, \\ Berkeley, California; Departamento de Parasitología, Centro Nacional de Diagnóstico y Referencia, Ministerio de Salud, \\ Managua, Nicaragua; Dirección de Epidemiología, Ministerio de Salud, Leon, Nicaragua; Hospital Dermatológico Nacional, \\ Managua, Nicaragua; Programa Nacional de Control de Leishmaniasis, Ministerio de Salud, Managua, Nicaragua
}

\begin{abstract}
Leishmania chagasi, the causative agent of visceral leishmaniasis (VL) in the Americas, has recently been associated with atypical cutaneous leishmaniasis (ACL) in Central America; however, little comprehensive information about this disease is available. Clinical, epidemiologic, and parasitologic characteristics of 252 ACL cases and $44 \mathrm{VL}$ cases in Nicaragua were analyzed. Visceral leishmaniasis is primarily associated with malnourished children less than five years of age, whereas ACL is found predominantly in children greater than five years of age and young adults. Genetically similar parasites are associated with both disease manifestations. The sand fly Lutzomyia evansi, in addition to Lu. longipalpis, may be involved in transmission of L. chagasi to humans. Our results indicate that ACL is more prevalent than previously thought, affecting up to $10 \%$ of a local population. The fact that the same parasite appears to cause both ACL and the potentially fatal visceral disease suggests that the host immune response is critical in determining the outcome of L. chagasi infection. The public health implications of the widespread presence of L. chagasi are discussed.
\end{abstract}

Parasitic protozoa of the genus Leishmania are responsible for a group of diseases that constitute an important public health problem in tropical and subtropical regions worldwide. Leishmaniasis presents a wide spectrum of clinical manifestations, depending on the species of the parasite, the host immune response, and possibly factors in the saliva of the sand fly vector. ${ }^{1}$ In the Americas, L. chagasi causes subclinical infections and visceral leishmaniasis (VL), which is potentially fatal if untreated. ${ }^{2}$ Recently, L. chagasi has been reported to cause non-ulcerated cutaneous nodules, designated atypical cutaneous leishmaniasis (ACL) or non-ulcerated cutaneous leishmaniasis, in Honduras, Costa Rica, and El Salvador (Murillo L, unpublished data).,4 Parasites responsible for VL and ACL in Honduras appear genetically similar as determined by a variety of molecular biological assays, such as polymerase chain reaction (PCR)-schizodemes, random amplified polymorphic DNA (RAPD) PCR, and differential display analysis. ${ }^{2}$

Leishmania infantum, a closely-related parasite found in the Mediterranean basin, has also been reported to cause both visceral and cutaneous disease..$^{5-8}$ In this case, parasites responsible for the different disease outcomes appear to correlate with different genetic classifications (zymodemes). However, typically dermotropic strains of L. infantum are known to visceralize in immunocompromised patients with acquired immunodeficiency syndrome (AIDS). ${ }^{9-11}$

Visceral leishmaniasis associated with L. chagasi infection in Nicaragua has been reported since the early 1980s (Belli A, Darce M, Moran J, Palacios X, Valle S, Gonzalez A, unpublished data). ${ }^{12}$ In 1997, the first cases of ACL were identified, and the incidence has been increasing dramatically. Here we report the clinical, epidemiologic, entomologic, and parasitologic characteristics of VL and ACL in Nicaragua. Our data suggest that human L. chagasi infections are more prevalent than previously estimated and could constitute a significant problem in other areas where $L$. chagasi is endemic, particularly in immunocompromised hosts.

\section{MATERIALS AND METHODS}

Patients and clinical data. The patients in this study were referred to the Centro Nacional de Diagnóstico y Referencia, Ministry of Health (Managua, Nicaragua) from regional hospitals and health units. A diagnosis of VL was made when patients had fever, hepatosplenomegaly, and an IgG titer higher than $1 / 64$ by an indirect immunofluorescence test. Additional signs included anemia (hematocrit below 35\%), malnutrition (according to the classification of Gómez and others), ${ }^{13}$ leukopenia (leukocyte count below 4,000/ $\mathrm{mm}^{3}$ ), and weight loss (as determined by the guardian). The 44 cases of VL included in this study occurred over a period of 10 years (1987-1997).

Two hundred eleven of the ACL cases were referred from hospitals and health units, and 41 were identified through a complete house-to-house survey in the community of Apompua in Chontales. Atypical cutaneous leishmaniasis was defined by at least one of the following: 1) the presence of non-ulcerated cutaneous nodules that contained Leishmania parasites by microscopy, 2) the presence of such lesions containing Leishmania DNA as determined by Leishmania genus-specific PCR, or 3) the presence of characteristic lesion(s), a positive Montenegro skin test result, and a positive immunofluorescence assay result. These 252 cases of ACL were reported and confirmed between April 1997 and October 1998. The VL and ACL specimens from patients visiting hospitals or Ministry of Health clinics were obtained by hospital staff and/or Ministry of Health personnel for routine diagnostic procedures, which included clinical examination, Montenegro skin test, serology, microscopy smear, culture, and PCR analysis. Unused portions of the PCR samples were used for PCR-schizodeme analysis. As per standard procedure in active case detection activities by the National Leishmaniasis Control Program of the Nicaraguan Ministry of Health, informed consent was obtained by Ministry of Health personnel from participants in the houseto-house survey.

Parasites. Reference strains included in this study were 
as follows: L. chagasi (MHOM/BR/74/PP75), L. braziliensis (MHOM/BR/75/2903), and L. mexicana (MNYC/BZ/62/ M379), obtained from the London School of Tropical Medicine and Hygiene (London, United Kingdom), and Honduran L. chagasi isolates from ACL cases (MHOM/HN/88/ HN116, MHOM/HN/97/HN901) or from visceral cases (MHOM/HN/87/HN029, MHOM/HN/97/HN905), kindly donated by Dr. Carlos Ponce (Laboratorio Central, Ministerio de Salud Pública, Tegucigalpa, Honduras).

Laboratory methods. Serum specimens from VL and ACL cases were analyzed by an indirect immunofluorescent antibody test (IFAT) using solid-phase L. chagasi promastigote antigen to capture IgG antibodies. ${ }^{14}$ Serum titers greater than or equal to $1 / 64$ were considered positive. The Montenegro skin test was performed on patients suspected of ACL by intradermal injection in the forearm of $0.1 \mathrm{ml}$ of a saline solution containing $5 \times 10^{5}$ killed L. chagasi promastigotes and $0.01 \%$ merthiolate. The area of induration was measured $48 \mathrm{hr}$ post-injection, and a diameter greater than or equal to $5 \mathrm{~mm}$ was considered positive. Smears were prepared from lesion scrapings, fixed with methanol, and stained with Giemsa for microscopy. Small elliptical incisional biopsies were taken using a sterile scalpel from facial lesions of the first seven patients presenting non-ulcerated cutaneous lesions who were seen by local dermatologists and later referred to our group. These skin biopsies were fixed in $10 \%$ formalin, embedded in paraffin, sectioned, and stained with hematoxylin and eosin for histologic analysis. ${ }^{15}$ Once the etiology of these lesions was confirmed and diagnosis shown to require only dermal scrapings, biopsies for histologic sections were suspended.

Fine-needle lesion aspirates were cultured in at least one of the following media: Schneider's Drosophila medium (Gibco-BRL, Gaithersburg, MD) supplemented with $10 \%$ fetal bovine serum (FBS; Gemini Bioproducts, Inc., Calabasas, CA), tryptic soy agar (TSA; Difco, Detroit, MI) containing $10 \%$ defibrinated rabbit blood overlaid with Schneider's Drosophila medium, TSA overlaid with a modified minimal essential liquid medium (HOSMEM II) ${ }^{14}$ supplemented with $10 \%$ FBS, and Senekjie ${ }^{16}$ medium overlaid with Locke's solution.

Entomologic methods. Phlebotomine sand flies were captured using Center for Disease Control and Prevention (CDC) light traps ${ }^{17}$ placed in the vicinity of houses of confirmed cases from dusk to dawn. The sand flies were transported in $70 \%$ ethanol, sorted, clarified with $20 \%$ potassium hydroxide, and speciated according to morphologic criteria.

Analysis by PCR. Dermal scrapings for PCR amplification were collected with a sterile wooden toothpick and placed in $100 \mu \mathrm{l}$ of $5 \% \mathrm{Chelex}^{\circledR} 100$ (Bio-Rad Laboratories, Hercules, CA) and boiled for 10 minutes. ${ }^{18}$ The DNA from peripheral blood mononuclear cells was prepared as in Belli and others. ${ }^{18}$ Amplification was conducted as described in Belli and others ${ }^{18}$ with primers $13 \mathrm{~A}$ and $13 \mathrm{~B}$, which target a region of the minicircle conserved in all Leishmania species. ${ }^{19}$ Parasites were characterized using 1) a multiplex PCR assay that targets the spliced leader RNA gene repeat (miniexon) and generates differently sized products for the three New World complexes, ${ }^{20}$ and 2 ) a $L$. donovani complex-specific PCR assay. ${ }^{21}$ Entire minicircles were amplified with the primer pair described in Smyth and others ${ }^{22}$ or primers $13 \mathrm{Y}^{19}$

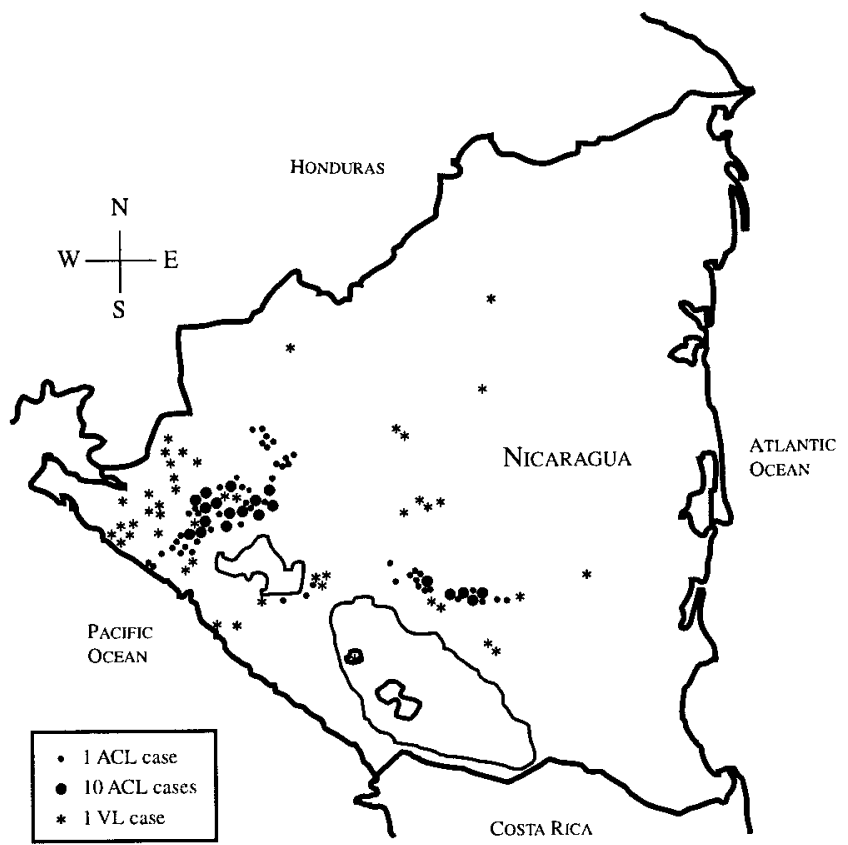

FIGURE 1. Map of Nicaragua showing the location of cases of atypical cutaneous leishmaniasis (ACL) and visceral leishmaniasis (VL).

and $\mathrm{LiR},{ }^{2}$ which target the conserved region of the minicircle DNA. The products were gel purified using the QIAquick (iiN) gel extraction method (QIAGEN Inc., Valencia, CA) and digested with the restriction enzyme Hae III (New England Biolabs, Inc., Beverly, MA).

Statistical analysis. The Wilcoxon rank sum test ${ }^{23}$ was used to compare the means of VL and ACL patient populations, while chi-square analysis was used to compare the distribution of phlebotomine populations in two geographic areas.

\section{RESULTS}

Visceral leishmaniasis. All 44 cases of VL were characterized by fever, hepatosplenomegaly, pancytopenia, and increased immunoglobulin levels, while 39 (89\%) cases presented with anemia, $25(57 \%)$ with leukopenia, $36(82 \%)$ with malnutrition, and $23(52 \%)$ with weight loss. All cases were confirmed as positive by immunofluorescence, as determined by high titers of serum antibodies specific to $L$. chagasi antigen. The age of patients ranged from nine months to 32 years (mean $=3.8$ years); 37 of the patients $(84 \%)$ were four years of age or younger. Eighteen $(41 \%)$ of the 44 cases came from the Department of Chinandega, $4(9 \%)$ from León, 6 (14\%) from Managua, 5 (11\%) from Chontales, and the other $11(25 \%)$ from five other departments (Figure 1). These regions are characteristically semiarid and below 200 meters above sea level.

Atypical cutaneous leishmaniasis. Atypical cutaneous leishmaniasis cases $(n=252)$ presented with cutaneous nodules that ranged in size from 3 to $30 \mathrm{~mm}$. In 209 cases $(83 \%)$, lesions were surrounded by an area of depigmentation. Histologic sections from skin biopsies demonstrated granulomatous infiltration $(n=7)$. The lesions were gener- 
ally few in number $($ mean $=2.5)$, with $84 \%$ of the patients presenting with three or less nodules; $190(75 \%)$ of the patients had lesions on the face, $62(25 \%)$ on the arms, 46 (18\%) on the legs, and $27(11 \%)$ on the torso. The lesions were reportedly present for 1 month to 15 years, with an average duration of 2.3 years. Forty-one patients reported the existence of these lesions for more than 5 years. No fever or hepatosplenomegaly was observed. Of the 252 ACL cases, $188(75 \%)$ were between the ages of 5 and 20 . The mean age of the ACL patients was 14.9 years, significantly different from that of the visceral cases, which was 3.8 years $(P$ $<0.01$, by Wilcoxon rank sum test). Of the confirmed ACL patients, $185(73 \%)$ came from the Department of León, 61 (24\%) from Chontales, 5 (2\%) from Managua, and one $(0.4 \%)$ from Granada (Figure 1). Most of the ACL cases in the Department of Chontales were from the community of Apompua, where no visceral disease has ever been reported. A complete house-to-house survey of 100 houses in this community revealed a prevalence of confirmed ACL of $9.6 \%$ among its population of 427 . This region is ecologically similar to areas where VL is endemic.

A number of diagnostic tests were performed. One hundred twenty $(79 \%)$ of 151 cases demonstrated a positive Montenegro skin test result, and 171 (84\%) of 203 had elevated $(>1 / 64)$ titers of specific antibodies to L. chagasi. Dermal scrapings from $53(23 \%)$ of 232 ACL patients revealed amastigotes by microscopy, although in most cases, parasites were few in number. Two hundred five $(85 \%)$ of 242 cases presented lesions containing Leishmania DNA, as determined by PCR assays. Efforts were made to isolate Leishmania in culture from 143 lesion aspirates in four different culture media; however, only six attempts (4\%) were successful, all in Schneider's Drosophila media supplemented with $10 \%$ FBS over TSA containing $10 \%$ rabbit blood.

Entomologic data. Sandfly collections were conducted in the proximity of reported leishmaniasis cases using CDC light traps, and the species of the captured sand flies were identified. In the Pacific region, including the Departments of Leon and Chinandega where VL cases predominate, 4, 852 phlebotomine specimens were collected over a period of 10 years. Seventy-seven percent of the sand flies were found to be Lutzomyia longipalpis, and $16 \%$ were classified as $L u$. evansi. Recent collections from communities reporting ACL in Chontales Department in Central Nicaragua revealed $47 \%$ Lu. longipalpis and $46 \%$ Lu. evansi $(\mathrm{n}=1,415)$. The relative numbers of Lu. longipalpis and Lu. evansi collected in these two regions demonstrate differences that are statistically significant $(P<0.001)$. Of the total number of captured phlebotomine sand flies $(\mathrm{n}=6,267), 93 \%$ were either $\mathrm{Lu}$. longipalpis or Lu. evansi.

PCR results. Peripheral blood mononuclear cells from 9 patients with VL were subjected to PCR amplification using primers specific to the genus Leishmania, and 7 generated products of the expected size of 120 basepairs (e.g., Figure 2, lanes 3 and 4). Crude preparations of dermal scrapings from ACL lesions were subjected to PCR amplification with genus-specific primers (Figure 2, lanes 5-9). Of 242 cases, $205(85 \%)$ were positive by the PCR. To characterize the parasite causing ACL and VL, several specimens were analyzed using a multiplex PCR assay that generates products of different sizes for each New World Leishmania complex.

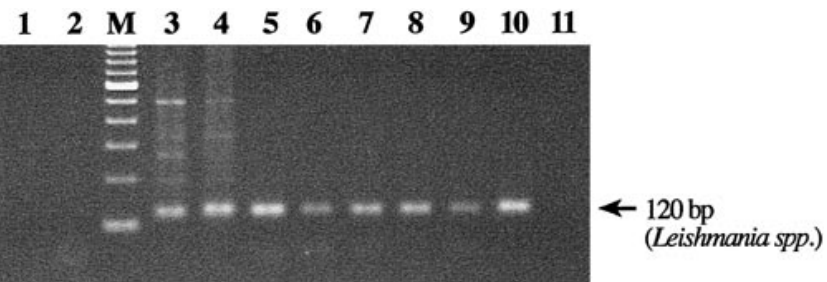

FIGURE 2. Diagnosis of atypical cutaneous leishmaniasis (ACL) and visceral leishmaniasis (VL) patients using a Leishmania genusspecific polymerase chain reaction (PCR). Dermal scrapings from cutaneous lesions were boiled for $10 \mathrm{~min}$ in a solution of $5 \%$ Chelex $100^{\circledR}$ and amplified using Leishmania genus-specific primers. Lane 1 , negative control (water); lane 2, dermal scraping from a nonleishmanial cutaneous lesion; lane M, 100-basepair (bp) ladder (lowest fragment shown, $100 \mathrm{bp}$ ); lanes 3 and 4, peripheral blood mononuclear cells from VL patients 1 and 2; lanes 5-9, dermal scrapings from ACL patients 1-5; lane 10, L. chagasi (MHOM/BR/ 74/PP75); lane 11, L. braziliensis (MHOM/BR/75/2903). Expected product size, $120 \mathrm{bp}$.

The samples yielded products within the size range expected for members of the L. donovani complex (e.g., Figure 3, lane 6). Cultured isolates from 6 ACL cases and 2 visceral leishmaniasis cases were also examined by both the multiplex assay and an L. donovani-specific minicircle-based PCR assay. Again, products of the expected size were obtained (Figure 3, lanes 7-14).

Schizodeme results. Kinetoplast DNA was amplified directly from clinical samples and from cultured isolates using two different sets of primers that amplify the variable region of the Leishmania minicircle. The resulting products were purified and digested with Hae III. Similar results were obtained with both primer sets. The patterns generated from parasites causing ACL $(n=6)$ and from parasites isolated from visceral cases in Nicaragua $(n=2)$ were similar, as shown in Figure 4, lanes 1-5. This same result was obtained with parasites from ACL and VL patients in Honduras, which also demonstrated similar patterns (Figure 4, lanes 69). However, L. chagasi isolates from both Nicaraguan VL and ACL cases generated a pattern that was distinct from that obtained with the Honduran parasites (Figure 4, lanes 1-9). Restriction digests of amplified minicircles from four of the Nicaraguan ACL isolates (e.g. Figure 4, lanes 4 and 5) generated a pattern that differed slightly from the schizodeme pattern characteristic of the other Nicaraguan ACL and VL isolates examined to date (e.g., Figure 4, lanes 1-3).

\section{DISCUSSION}

We report here that $L$. chagasi causes both visceral and cutaneous disease in Nicaragua, and describe clinical and epidemiologic characteristics of atypical $L$. chagasi infections. We found that the parasites responsible for both visceral and cutaneous disease appear to be similar. Since the clinical manifestation of leishmaniasis depends on both parasite and host factors, this finding suggests that the outcome of L. chagasi infection may be in large part determined by the host immune response. We believe that atypical cutaneous leishmaniasis (ACL) is an intermediate form in the spectrum from subclinical $L$. chagasi infections to visceral disease. We find that ACL is significantly more widespread than 


\section{$\begin{array}{llllllllllllllllllllll}1 & 2 & 3 & 4 & 5 & 6 & 7 & 8 & 9 & 10 & M & 11 & 12 & 13 & 14 & 15 & 16 & 17 & 18\end{array}$}

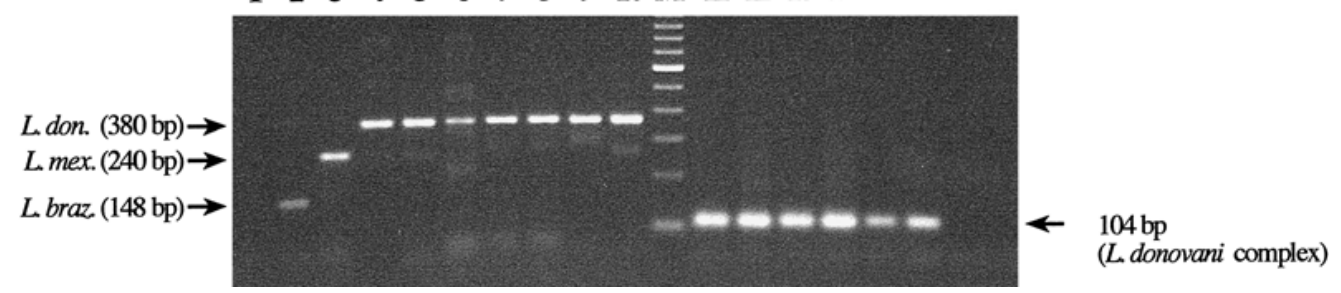

FIGURE 3. Characterization of Nicaraguan Leishmania chagasi isolates by the polymerase chain reaction (PCR). Lanes 1-10, samples amplified using a New World Leishmania multiplex PCR; expected product sizes: L. donovani (don.) complex, 351-397 basepairs (bp); L. mexicana (mex.) complex, 218-240 bp; and L. braziliensis (braz.) complex, 146-149 bp. Lane 1, negative control (water); lane 2, L. braziliensis (MHOM/BR/75/2903); lane 3, L. mexicana (MNYC/BZ/62/M379); lane 4, L. chagasi (MHOM/BR/74/PP75); lane 5, Honduran atypical cutaneous leishmaniasis (ACL) isolate (MHOM/HN/88/HN116); lane 6, dermal scraping from Nicaraguan ACL patient LC9786; lane 7, isolate from Nicaraguan ACL patient (MHOM/NI/97/LC9786); lane 8, isolate from Nicaraguan ACL patient (MHOM/NI/98/LC9812); lane 9, isolate from Nicaraguan visceral leishmaniasis (VL) patient (MHOM/NI/92/LV1); lane 10, isolate from Nicaraguan VL patient (MHOM/NI/92/LV2); lane M, 100-bp ladder (lowest fragment shown, 100 bp). Lanes 11-18, samples amplified with $L$. donovani complex-specific primers; expected product size 104 bp. Lane 11, isolate from Nicaraguan ACL patient (MHOM/NI/97/LC9786); lane 12, isolate from Nicaraguan ACL patient (MHOM/NI/98/LC9812); lane 13, isolate from Nicaraguan VL patient (MHOM/NI/92/LV1); lane 14, isolate from Nicaraguan VL patient (MHOM/NI/92/LV2); lane 15, Honduran ACL isolate (MHOM/HN/88/HN116); lane 16, L. chagasi (MHOM/BR/74/PP75); lane 17, L. braziliensis (MHOM/BR/75/2903); lane 18, negative control (water).

previously thought and believe that the public health implications of this phenomenon must be considered.

Atypical cutaneous leishmaniasis cases occur in semi-arid areas typical of the Central and Pacific regions of Nicaragua (Figure 1) and other Central American countries. ${ }^{3,4}$ Our data from a household survey in an endemic community revealed a prevalence of approximately $10 \%$. This is consistent with the high numbers of cases reported in affected areas in Honduras (Ponce $\mathrm{C}$, unpublished data) and Costa Rica. ${ }^{4}$ Given the apparent high frequency of ACL among affected populations, as awareness of ACL increases, these case reports could reach significant proportions in other regions where $L$. chagasi is endemic (e.g., Central America, Brazil, Colombia).

In this retrospective study, we report 44 cases of classical VL caused by $L$. chagasi that were seen at the Centro $\mathrm{Na}-$ cional de Diagnóstico y Referencia in Managua over the last 10 years and 252 cases of ACL that were diagnosed within

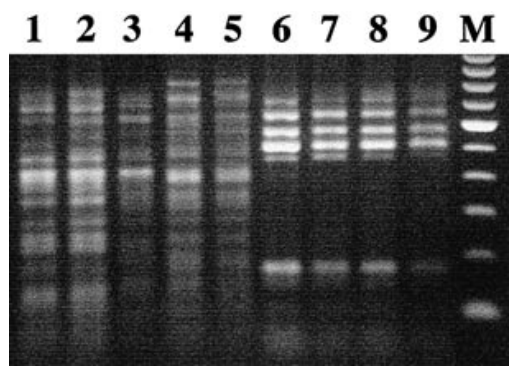

FIGURE 4. Polymerase chain reaction (PCR)-schizodeme analysis of parasites isolated from Nicaraguan and Honduran patients with atypical cutaneous leishmaniasis (ACL) and visceral leishmaniasis (VL). Digestion of PCR-amplified minicircle DNA with Hae III. Lane 1, isolate from Nicaraguan VL patient (MHOM/NI/92/LV1); lane 2, isolate from Nicaraguan VL patient (MHOM/NI/92/LV2); lane 3, isolate from Nicaraguan ACL patient (MHOM/NI/98/ LCA98294); lane 4, isolate from Nicaraguan ACL patient (MHOM/ NI/98/LCA98292); lane 5, isolate from Nicaraguan ACL patient (MHOM/NI/98/LCA98284); lane 6, Honduran ACL isolate (MHOM/HN/88/HN116); lane 7, Honduran ACL isolate (MHOM/ HN/97/HN901); lane 8, Honduran VL isolate (MHOM/HN/87/ HN029); Honduran VL isolate (MHOM/HN/97/HN905); Lane M, 100-basepair (bp)-ladder (lowest fragment shown, 100 bp). the last year and a half. This upsurge in reports of ACL probably reflects diagnostic bias due to increased awareness, and not necessarily a change in disease incidence. Atypical cutaneous leishmaniasis is characterized by the presence of cutaneous nodules found on the face and extremities, often surrounded by an area of depigmentation. There is no detectable fever or hepatosplenomegaly, which are characteristic of VL.

The ability of L. chagasi to cause both visceral and cutaneous disease could be determined by a number of factors, including the status of the host immune system, the genetic identity and characteristics of the parasite, and the sand fly responsible for its transmission. The immune response is influenced by the nutritional state of the host, which in turn is a reflection of socioeconomic status. Interestingly, a number of the ACL cases in Nicaragua come from regions where both pathologies (ACL and VL) are reported (e.g., Malpaisillo, Department of Leon), whereas other ACL cases come from areas with no history of VL (e.g., Apompua, Department of Chontales) (Figure 1). The areas where visceral and cutaneous disease co-exist appear to be more economically depressed and have higher levels of malnutrition than communities reporting only ACL (Belli A, unpublished data). This is consistent with earlier reports that identified malnutrition as a risk factor for the development of visceral disease. ${ }^{24}$ Another notable observation is the difference in age between visceral cases (mean $=3.8$ years) and ACL cases (mean $=14.9$ years; $P<0.01)$; this may reflect differential disease susceptibility related to maturation of the immune system. Studies are currently underway to investigate the cellular immune response of ACL patients.

Another factor that could influence severity of disease is the parasite itself. The closely-related Old World parasite $L$. infantum has been associated with both cutaneous and visceral disease in the Mediterranean basin, and dermotropic $L$. infantum strains appear to belong to a different zymodeme than viscerotropic strains when analyzed using the high-resolution technique multilocus enzyme electrophoresis. ${ }^{5-8}$ However, our preliminary results indicate that $L$. chagasi parasites responsible for VL and ACL in Nicaragua are ge- 
netically similar, according to the patterns generated by restriction enzyme digestion of PCR-amplified minicircles (Figure 4). This is consistent with reports that L. chagasi strains isolated from either visceral or ACL patients in neighboring Honduras display similar PCR-schizodeme and RAPD patterns. ${ }^{2}$ A small degree of clonal heterogeneity was observed among the Nicaraguan parasites, since several ACL isolates generated a pattern that differed slightly from the pattern shared by the other ACL and VL strains.

Interestingly, the PCR-schizodeme patterns of Nicarguan and Honduran L. chagasi strains are distinct (Figure 4). ${ }^{2}$ Since this schizodeme analysis is PCR-based, it is possible that different minicircle classes are being amplified from parasites from the two countries; even if this is the case, this would reflect a certain genetic distinction. Most significantly, isolates from the two countries exhibit distinct biologic behaviors. The Honduran strains can be readily isolated in culture, while the Nicaraguan parasites are extremely fastidious and difficult to grow in culture-a characteristic for which dermotropic L. infantum and cutaneous Costa Rican L. chagasi strains are notorious., ${ }^{45,26}$ These observations may indicate that clonal populations of $L$. chagasi are circulating in Nicaragua that are genetically distinct from the parasite population found in Honduras, both of which are capable of causing the two pathologies.

The species of the transmitting sand fly could also play a role in determining the clinical outcome of L. chagasi infection. It has been suggested that factors in the saliva of the sand fly, such as the vasoactive peptide maxadilan, can influence tissue tropism. ${ }^{27,28}$ Lutzomyia longipalpis is considered to be the primary vector for transmission of L. chagasi associated with visceral disease. ${ }^{29}$ Over a 10-year period, we found that $\mathrm{Lu}$. longipalpis was the predominant phlebotomine species in foci of active transmission of visceral leishmaniasis, while $L u$. evansi, a secondary vector for $L$. chagasi, represented only a small proportion of the sand flies collected. In contrast, recent investigation of several areas reporting only ACL activity revealed a greater proportion of Lu. evansi, equal to that of Lu. longipalpis. Although these differences are statistically significant, the relative numbers of Lu. longipalpis and Lu. evansi collected may be affected by seasonal variation, a possibility that we are currently investigating. These preliminary data suggest that $L u$. evansi as well as Lu. longipalpis may be involved in transmission of L. chagasi. In addition, the distinct species of Lutzomyia could influence the different clinical manifestations of VL and ACL. Confirmation of the vector responsible for transmission of L. chagasi in foci of ACL is under further examination.

The public health implications of widespread L. chagasi infections in Central and South America are manifold. First, parasites that cause ACL appear to be genetically very similar to those responsible for visceral leishmaniasis. If this is the case, this poses the risk that cutaneous infection may visceralize upon deterioration of the immune status of the host, since dermotropic variants of $L$. infantum are known to visceralize in immunocompromised patients with AIDS. ${ }^{6,9-11}$ Second, ACL cases may serve as a reservoir for transmission of L. chagasi infections, which could lead to visceral disease in subsequent hosts. If so, treatment of ACL cases could potentially serve as a preventive measure to- wards reducing the incidence of VL. In fact, this phenomenon has been observed in Honduras, where aggressive treatment of ACL in various communities led to an apparent decrease in visceral cases (Ponce $\mathrm{C}$, unpublished data). To explore the possibility of ACL cases acting as a reservoir, studies are underway in Nicaragua to investigate whether ACL leads to a systemic infection with $L$. chagasi. Third, cutaneous lesions associated with ACL are often misdiagnosed (e.g., as cutaneous tuberculosis, sarcoidosis, or lupus vulgaris) and treated inappropriately at high cost. Last, ACL may serve as a sentinel clinical marker for the possible occurrence of VL in a community, with the incidence of VL influenced by other factors, such as malnutrition in children or immunosuppression. It is likely that ACL is being overlooked to a large extent and that L. chagasi infections are more prevalent than previously thought.

Acknowledgments: We are grateful to Alcides Gonzalez, Director of the Centro Nacional de Diagnóstico y Referencia, for support, and to the health personnel and the community of Apompua for their collaboration. We thank Michael Diamond and Lee W. Riley for helpful editorial comments.

Financial support: This work was supported by the NIH Fogarty International Center (D43 TW00905), International Scientific Cooperation Program of the European Union (CI1 T92-00 60), the John D. and Catherine T. MacArthur Foundation, and the Fondation Père Damien.

Authors' addresses: Alejandro Belli and Eva Harris, Infectious Diseases Unit, Division of Public Health Biology and Epidemiology, School of Public Health, University of California, Berkeley, 140 Warren Hall, Berkeley, CA 94720-7360. David García, Dirección de Epidemiología, Ministerio de Salud, Leon, Nicaragua. Xiomara Palacios, Betzabé Rodriguez, Sonia Valle and Elsa Videa, Departamento de Parasitología, Centro Nacional de Diagnóstico y Referencia, Ministerio de Salud, Apartado Postal 2900, Managua, Nicaragua. Ena Tinoco, Hospital Dermatológico Nacional, Managua, Nicaragua. Francicsa Marín, Programa Nacional de Control de Leishmaniasis, Ministerio de Salud, Concepción Palacios, Managua, Nicaragua.

Reprint requests: Eva Harris, Infectious Diseases Unit, Division of Public Health Biology and Epidemiology, School of Public Health, University of California, Berkeley, 140 Warren Hall, Berkeley, CA 94720-7360.

\section{REFERENCES}

1. Grimaldi Jr G, Tesh RB, 1993. Leishmaniasis of the New World: current concepts and implications for future research. Clin Microbiol Rev 6: 230-250.

2. Noyes H, Chance M, Ponce C, Ponce E, Maingon R, 1997. Leishmania chagasi: genotypically similar parasites from Honduras cause both visceral and cutaneous leishmaniasis in humans. Exp Parasitol 85: 264-273.

3. Ponce C, Ponce E, Morrison A, Cruz A, Kreutzer R, McMahonPratt D, Neva F, 1991. Leishmania donovani chagasi: new clinical variant of cutaneous leishmaniasis in Honduras. Lancet 337: $67-70$.

4. Zeledón R, Hidalgo H, Víquez A, Urbina A, 1989. Atypical cutaneous leishmaniasis in a semiarid region of north-west Costa Rica. Trans R Soc Trop Med Hyg 83: 786.

5. Bettini S, Gramiccia M, Gradoni L, Biggio P, Loi R, Cottoni F, Pau M, Atzeni MC, 1990. Leishmaniasis in Sardinia. IV. Epidemiological appraisal of cutaneous leishmaniasis and biochemical characterization of isolates. J Trop Med Hyg 93: 262-269.

6. Gramiccia M, Gradoni L, Troiani M, 1992. HIV-Leishmania coinfections in Italy. Isoenzyme characterization of Leishmania 
causing visceral leishmaniasis in HIV patients. Trans $R$ Soc Trop Med Hyg 86:161-163.

7. Belazzoug S, Lanotte G, Maazoun R, Pratlong F, Rioux JA, 1985. A new enzymatic variant of Leishmania infantum (Nicolle 1908) agent of cutaneous leishmaniasis in northern Algeria. Ann Parasitol Hum Comp 60: 1-3.

8. Marty P, Pratlong F, Marcelet B, Adda A, Le Fichoux Y, 1994. Leishmania infantum variant MON-24 isolated from a cutaneous lesion acquired in the suburban area of Nice. Parasite 1: $175-176$.

9. Alvar J, Canavate C, Gutierrez-Solar B, Jimenez M, Laguna F, Lopez-Velez R, Molina R, Moreno J, 1997. Leishmania and human immunodeficiency virus coinfection: the first 10 years. Clin Microbiol Rev 10: 298-319.

10. Gramiccia M, Gradoni L, Troiani M, 1995. Heterogeneity among zymodemes of Leishmania infantum from HIV-positive patients with visceral leishmaniasis in south Italy. FEMS Microbiol Lett 128: 33-38.

11. Marty P, Fuzibet FG, Pratlong F, Quinsat D, Gari-Toussaint M, Dor JF, Le Fichoux Y, Rioux JA, 1991. Visceral leishmaniasis caused by a dermotropic strain of Leishmania infantum in an AIDS patient. Bull Soc Pathol Exot 84: 365-367.

12. Duarte Z, Munguia J, Orozco M, Gantier JC, 1994. First report of visceral leishmaniasis in Nicaragua. Trans $R$ Soc Trop Med Hyg 88: 38.

13. Gómez F, Ramos R, Frenk S, Cravioto J, Chavez R, Vasquez J, 1956. Mortality and second and third degree malnutrition. $J$ Trop Pediatr 2: 77-83.

14. Berens RL, Marr JJ, 1978. An easily prepared defined medium for cultivation of Leishmania donovani promastigotes. J Parasitol 64: 160.

15. Luna LG, ed., 1968. Manual of Histologic Staining Methods of the Armed Forces Institute of Pathology. Third edition. New York: McGraw-Hill Book Co., 38-39.

16. Senekjie H, 1943. Biochemical reactions, culture characteristics and growth requirements of Trypanosoma cruzi. Am J Trop Med Hyg 23: 523-531.

17. Sudia WD, Chamberlain WD, 1962. Battery-operated light trap, an improved model. Mosq News 22: 126-129.

18. Belli A, Rodriguez B, Avilés H, Harris E, 1998. Simplified PCR detection of New World Leishmania from clinical specimens Am J Trop Med Hyg 58: 102-109.

19. Rodgers MR, Popper SJ, Wirth DF, 1990. Amplification of kinetoplast DNA as a tool in the detection and diagnosis of Leishmania. Exp Parasitol 71: 267-275.

20. Harris E, Kropp G, Belli A, Rodriguez B, Agabian N, 1998. Single-step multiplex PCR assay for characterization of New World Leishmania complexes. J Clin Microbiol 36: 19891995.

21. Piarroux R, Azaiez R, Lossi AM, Reynier P, Muscatelli F, Gambarelli F, Fontes M, Dumon H, Quilici M, 1993. Isolation and characterization of a repetitive DNA sequence from Leishmania infantum: development of a visceral leishmaniasis polymerase chain reaction. Am J Trop Med Hyg 49: 364-369.

22. Smyth AJ, Ghosh A, Hassan MQ, de Brujin MHL, Adhya S, Mallik KK, Barker DC, 1992. Rapid and sensitive detection of Leishmania kinetoplast DNA from spleen and blood samples of kala-azar patients. Parasitology 105: 183-192.

23. Armitage P, Berry G, 1994. Statistical Methods in Medical Research. Third edition. Oxford: Blackwell Scientific Publications, 453-460.

24. Cerf BJ, Jones TC, Badaro R, Sampaio D, Teixeira R, Johnson $\mathrm{Jr}$ WD, 1987. Malnutrition as a risk factor for severe visceral leishmaniasis. J Infect Dis 156: 1030-1033.

25. Ben-Ismail R, Smith DF, Ready PD, Ayadi A, Gramiccia M, Ben-Osman A, Ben-Raehid MS, 1992. Sporadic cutaneous leishmaniasis in north Tunisia: identification of the causative agent as Leishmania infantum by the use of a diagnostic deoxyribonucleic acid probe. Trans $R$ Soc Trop Med Hyg 86 . 508-510.

26. Gramiccia M, Gradoni L, Pozio E, 1987. Leishmania infantum sensu lato as an agent of cutaneous leishmaniasis in Abruzzi region (Italy). Trans $R$ Soc Trop Med Hyg 81: 235-237.

27. Warburg A, Saraiva E, Lanzaro GC, Titus RG, Neva F, 1994 Saliva of Lutzomyia longipalpis sibling species differs in its composition and capacity to enhance leishmaniasis. Philos Trans $R$ Soc Lond B Biol Sci 345: 223-230.

28. Lanzaro WC, Warburg A, 1995. Genetic variability in phlebotomine sandflies: possible implications for leishmaniasis epidemiology. Parasitol Today 11: 151-154.

29. Zeledón R, 1985. Leishmaniasis in North America, Central America and the Caribbean islands. Chang KP, Bray RS, eds. Leishmaniasis. Amsterdam: Elsevier, 313-351. 